\title{
Piezoelectric Response to Coherent Longitudinal and Transverse Acoustic Phonons in a Semiconductor Schottky Diode
}

\author{
D. Srikanthreddy, ${ }^{1}$ B. A. Glavin, ${ }^{2}$ C. L. Poyser, ${ }^{1, *}$ M. Henini, ${ }^{1}$ D. Lehmann, ${ }^{3}$ Cz. Jasiukiewicz, ${ }^{4}$ \\ A. V. Akimov, ${ }^{1}$ and A. J. Kent ${ }^{1}$ \\ ${ }^{1}$ School of Physics and Astronomy, University of Nottingham, \\ University Park, Nottingham NG7 2RD, United Kingdom \\ ${ }^{2}$ V.E. Lashkaryov Institute of Semiconductor Physics, National Academy of Sciences, Kiev 03028, Ukraine \\ ${ }^{3}$ Institute for Theoretical Physics, TU Dresden, D-01062 Dresden, Germany \\ ${ }^{4}$ Faculty of Mathematics and Applied Physics, Rzeszów University of Technology, \\ aleja Powstańców Warszawy 8, PL-35-959 Rzeszów, Poland \\ (Received 25 October 2016; published 13 February 2017)
}

\begin{abstract}
We study the generation of microwave electronic signals by pumping a (311) GaAs Schottky diode with compressive and shear acoustic phonons, generated by the femtosecond optical excitation of an $\mathrm{Al}$ film transducer and mode conversion at the Al-GaAs interface. They propagate through the substrate and arrive at the Schottky device on the opposite surface, where they induce a microwave electronic signal. The arrival time, the amplitude, and the polarity of the signals depend on the phonon mode. A theoretical analysis is made of the polarity of the experimental signals. This analysis includes the piezoelectric and deformation potential mechanisms of electron-phonon interaction in a Schottky contact and shows that the piezoelectric mechanism is dominant for both transverse and longitudinal modes with frequencies below 250 and $70 \mathrm{GHz}$, respectively.
\end{abstract}

DOI: 10.1103/PhysRevApplied.7.024014

\section{INTRODUCTION}

The transduction of radio-frequency electrical signals to acoustic waves and back again is a technique that is widely exploited in communication technology: for example, in surface and bulk acoustic-wave filters, resonators, and delay lines. Such devices exploit the piezoelectric (PE) transformation of the lattice strain to the polarization $\mathbf{P}$ and back. In addition to these established applications, the PE effect has been utilized in the emerging research fields of nanotechnology, e.g., fabrication of nanogenerators [1] manipulations by strain of charge and spin transport at the surface of semiconductors [2], and control of electromechanical properties of nanolayers including graphene [3], all of which could also lead to the future development of alternative radio-frequency technologies.

Present acoustoelectric devices operate at frequencies up to a few gigahertz. There is a considerable research effort currently aimed at developing devices and systems for the full utilization for communications, imaging, and spectroscopy of the terahertz and subterahertz part of the

\footnotetext{
* Corresponding author. caroline.poyser@nottingham.ac.uk

Published by the American Physical Society under the terms of the Creative Commons Attribution 4.0 International license. Further distribution of this work must maintain attribution to the author(s) and the published article's title, journal citation, and DOI.
}

electromagnetic spectrum (ranging from approximately $100 \mathrm{GHz}$ to a few terahertz). Acoustoelectric devices for this part of the spectrum could be based on PE nanodevices having picosecond response times.

In recent decades, a number of experiments with coherent acoustic phonons have been performed in PE semiconductors and related nanolayers [4-11]. All of these works use femtosecond optical excitation to modulate the polarization in a PE sample by the generation of photocarriers, resulting in instantaneous stress. This stress generates terahertz coherent phonons (high-frequency acoustic waves), which, due to the PE field, modulate the optical properties in the same or a neighboring nanolayer for detecting the coherent phonons with the probe optical pulse. Importantly, the PE field enables the generation and detection of elastic shear perturbations which accompany transverse acoustic (TA) phonons [6-11]. The other electron-phonon interaction mechanism, the deformation potential (DP), does not couple electrons in an isotropic conduction band with shear perturbations. The theory of the generation and detection processes resulting from the PE effect has been reported in several publications $[7,12]$. In previous experiments with coherent phonons, the PE field in the sample has been measured indirectly by monitoring the optical properties. In this case, the PE effect is often masked or hidden by the DP mechanism [5], which produces an efficient optical response due to the modulation of the band-gap value [13].

It is appealing, therefore, to have an ultrafast broadband PE nanotransducer based on the PE effect, where 
high-frequency oscillating strain, which accompanies coherent phonons, is directly transformed to an electrical signal providing efficient response to both compressive and shear strain components. Such a device would also be a proof of principle for acoustoelectric devices operating at higher frequencies than previously achieved. Accomplishment of this challenging task is the goal of this work, which makes it fundamentally different from earlier works [14], where devices based on the DP mechanism of phonon-electric transformation have low efficiency and are insensitive to the TA phonons.

In this paper, we show that a Schottky contact fabricated on a low-symmetry surface of a semiconductor material which does not possess a center of inversion symmetry serves as an efficient PE transducer for both compressive and shear perturbations associated with coherent quasilongitudinal (QLA) and quasitransverse (QTA) phonons, respectively. Previously, PE transformation using Schottky contacts has been used in nanogenerators, but at low kilohertz frequencies [15]. In this work, we show that the PE transformation from QLA and QTA coherent phonons to an electrical signal in a Schottky diode works at microwave (gigahertz) frequencies, and the method is theoretically predicted to work up to subterahertz frequencies.

\section{EXPERIMENT AND RESULTS}

The experimental arrangement is shown in Fig. 1(a). A $1.5-\mu \mathrm{m}$-thick $n$-GaAs layer, doped with Si to a density of $n_{D}=1 \times 10^{17} \mathrm{~cm}^{-3}$, is grown by molecular beam epitaxy on a (311) $B$ surface of a semi-insulating GaAs substrate with the thickness $d=0.38 \mathrm{~mm}$. In addition, a 150-nmthick Au Schottky contact is deposited. The structure is processed into $100-\mu \mathrm{m}$-diameter and $0.8-\mu \mathrm{m}$-tall vertical device mesas, and a GeAuNiAu Ohmic contact is made to the $n$-GaAs layer [see the inset in Fig. 1(a)]. The sample is mounted on a holder incorporating a $50-\Omega$ microstrip line conductor and a microwave coaxial launcher for connection to the wide-bandwidth cryostat wiring. The main panel in Fig. 1(a) shows the Schottky diode's current-voltage $(I-V)$ characteristics at a temperature of $5 \mathrm{~K}$. The device turns on at a forward bias of $0.7 \mathrm{~V}$ and a reverse breakdown occurs at about $-5 \mathrm{~V}$.

The experiments are carried out in an optical cryostat at temperatures $T$ between 5 and $300 \mathrm{~K}$. A 100-nm-thick Al film deposited on the opposite side of the substrate to the Schottky device is excited by pulses from an amplified Ti: sapphire laser: pulse length, $80 \mathrm{fs}$; wavelength, $800 \mathrm{~nm}$; and repetition rate, $5 \mathrm{kHz}$. The laser is focused to a spot with a $50 \mu \mathrm{m}$ diameter, and the intensity on the $\mathrm{Al}$ film does not exceed $10 \mathrm{~mJ} \mathrm{~cm}^{-2}$. The optical excitation generated a compressive strain pulse in the Al film [16]. The injection of this strain pulse into the (311) GaAs substrate results in the generation of two coherent acoustic phonon wave packets propagating in the GaAs [17]: quasilongitudinal (QLA) with the displacement vector close to the normal [311], and
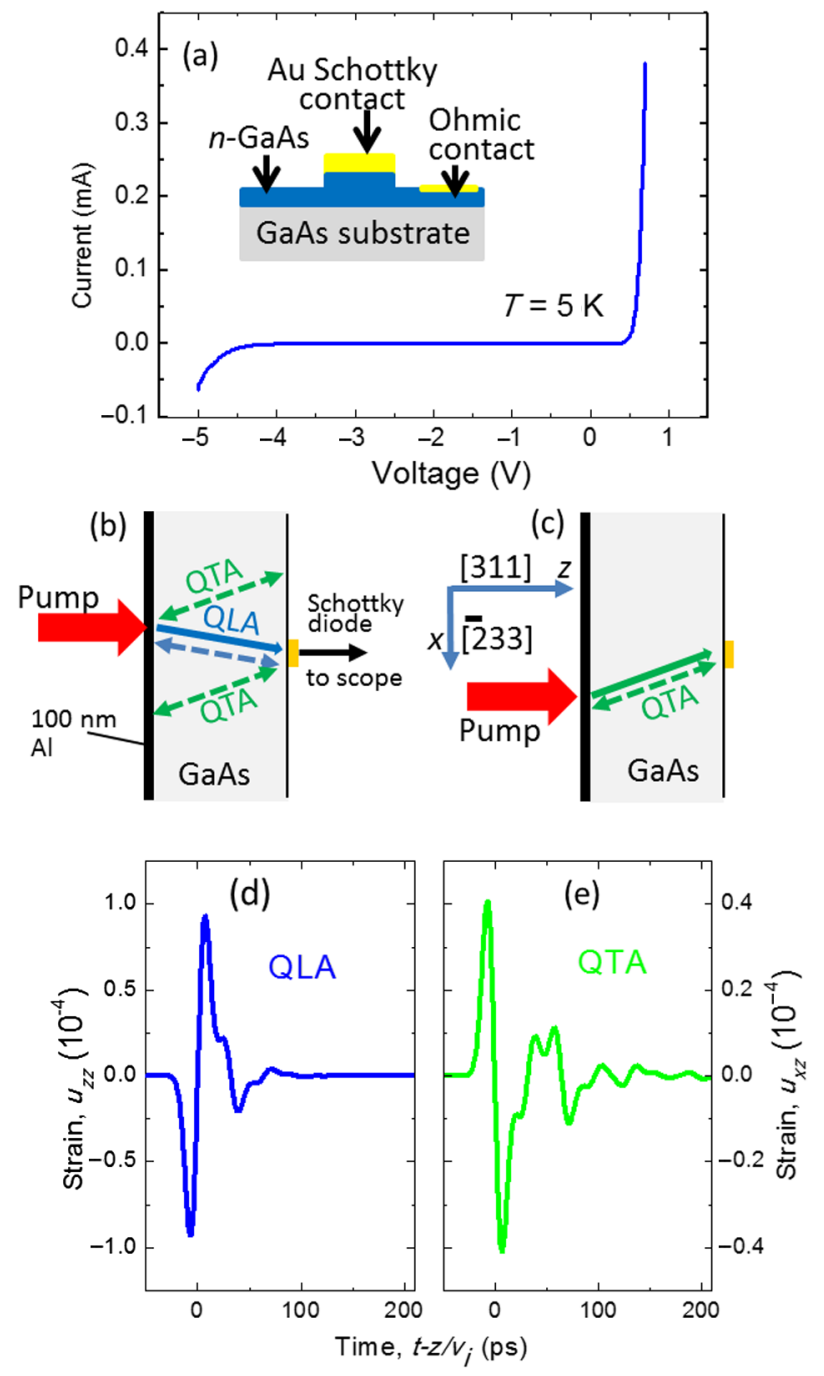

FIG. 1. (a) Current-voltage characteristics of the Schottky diode. Sample schematic shown in the inset. (b),(c) Schematic of the experiment, with coherent QLA and QTA phonons, respectively; the solid and dashed arrows indicate the direct and reflected phonon fluxes detected by the Schottky contact, respectively. (d),(e) Calculated strain waveforms for QLA and QTA phonons generated in an Al film with the pump fluence $W=1 \mathrm{~mJ} \mathrm{~cm}^{-2}$.

quasitransverse (QTA) with the displacement vector close to [233]. The third polarization, which corresponds to the pure TA mode, is not excited in this scheme. The wave vectors for QLA and QTA phonons, $\mathbf{q}_{\mathrm{QLA}}$ and $\mathbf{q}_{\mathrm{QTA}}$, respectively, parallel to [311]. However, owing to elastic anisotropy, their group velocities, $\mathbf{v}_{\mathrm{QLA}}^{g}$ and $\mathbf{v}_{\mathrm{QTA}}^{g}$, lying in the (011) plane, point at angles $\theta_{\mathrm{QLA}}$ and $\theta_{\mathrm{QTA}}$ to [311], as shown in Figs. 1(b) and 1(c), respectively. The components of the group velocity parallel to [311] are equal to the corresponding phase velocities $v_{\mathrm{QLA}}$ and $v_{\mathrm{QTA}}$. Solving the elastic equations with the elastic constants for GaAs [18], we obtain $v_{\mathrm{QLA}}=5.1 \mathrm{~km} \mathrm{~s}^{-1}, \quad v_{\mathrm{QTA}}=2.9 \mathrm{~km} \mathrm{~s}^{-1}, \quad \theta_{\mathrm{QLA}}=11.8^{\circ}$, and $\theta_{\mathrm{QTA}}=-22.5^{\circ}$. The polarizations are found to be 
$\mathbf{e}_{\mathrm{QLA}}=\left(u_{0}, 0, \sqrt{1-u_{0}^{2}}\right)$, and $\mathbf{e}_{\mathrm{QTA}}=\left(\sqrt{1-u_{0}^{2}}, 0,-u_{0}\right)$, with $u_{0} \approx 0.165$ [19]. To describe quantitatively the evolution of the strain components of the pulses, it is convenient to introduce the shape functions $\eta_{i}\left(t-z / v_{i}\right)$, with index $i$ marking the pulse polarization according to $u_{z z}^{(i)}=e_{z}^{(i)} \eta_{i}\left(t-z / v_{i}\right), u_{x z}^{(i)}=\left(e_{x}^{(i)} / 2\right) \eta_{i}\left(t-z / v_{i}\right)$.

To detect the injected phonon fluxes, the excitation spots of the $\mathrm{Al}$ film are displaced relative to the device, as shown in Figs. 1(b) and 1(c) for QLA and QTA wave packets, respectively. The calculated waveforms of the QLA and QTA strain pulses propagating in the GaAs substrates for the pump excitation density of the $\mathrm{Al}$ films $W=1 \mathrm{~mJ} \mathrm{~cm}^{-1}$ are shown in Figs. 1(d) and 1(e), respectively [17]. These waveforms do not include the nonlinear acoustic properties of GaAs.

After the propagation through the GaAs substrate in the time $\Delta t_{i}=d / v_{i}$, the coherent phonons induce an electrical response $\delta V(t)$ in the Schottky contact monitored by a 12.5-GHz digitizing oscilloscope. The insets in Figs. 2(a) and in 2(b) show the temporal traces $\delta V(t)$ measured for QLA and QTA coherent phonons, respectively. The traces include several sharp spikes. The spike at $t=0$ corresponds to the response from the optical-pump breakthrough and other spikes, marked by vertical arrows, are due to coherent phonon wave packets. We attribute the second spikes at $t \approx 79$ ns [inset in Fig. 2(a)] and $t \approx 121$ ns [inset in Fig. 2(b)] respectively to the QLA and QTA phonons propagating directly from the Al film to the Schottky device [see the solid arrows in Figs. 1(b) and 1(c)], with sound velocities of $v_{\mathrm{QLA}}=5.5 \mathrm{~km} \mathrm{~s}^{-1}$ and $v_{\mathrm{QTA}}=3.1 \mathrm{~km} \mathrm{~s}^{-1}$, which are close to the calculated values. Later spikes correspond to the multiple reflections in the substrate without and with mode conversion, as indicated in

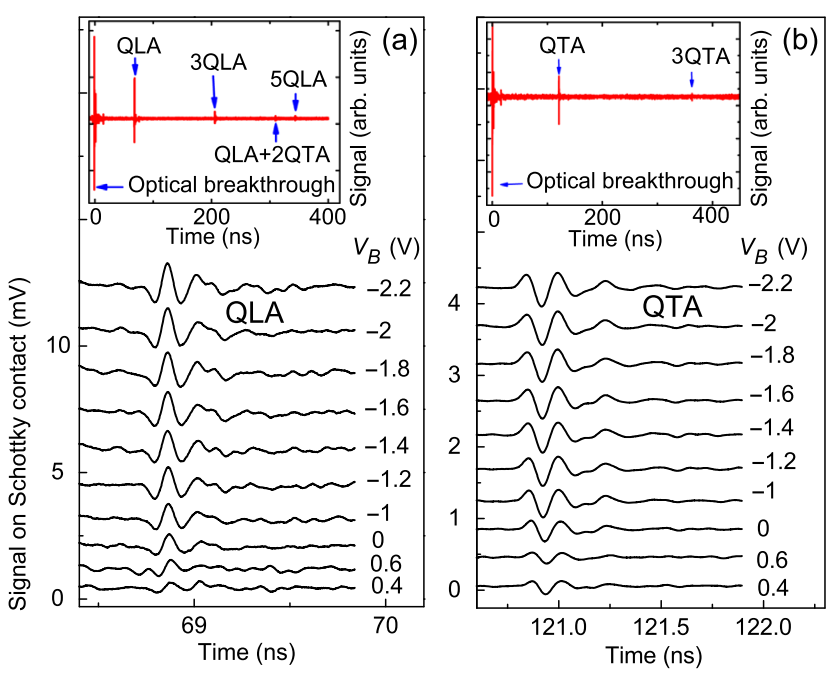

FIG. 2. Phonon-induced signals measured at pump fluence $W=4 \mathrm{~mJ} \mathrm{~cm}^{-2}$ for various bias voltages $V_{B}$ for (a) QLA and (b) QTA phonons. (Insets) QLA and QTA signals measured in a full temporal range.
Figs. 1(b) and 1(c) by dashed arrows. Mode conversion QLA $\leftrightarrow$ QTA may take place only at the GaAs-metal interface, which may happen at the side of the GaAs substrate with the $\mathrm{Al}$ film and at the Schottky Au contact.

The main panels in Figs. 2(a) and 2(b) show enlarged fragments of the electrical signals for QLA and QTA phonons, respectively, measured for several bias voltages $V_{b}$. The signals $\delta V(t)$ possess oscillatory behavior. It is important that the first peak in $\delta V(t)$ is negative for QLA and positive for QTA phonons. A detailed look at the enlarged fragments of $\delta V(t)$ reveals that the temporal positions $t_{m}$ of the first-peak minimum (for QLA) or maximum (for QTA) slightly moves to earlier times with an increase of reverse bias. This finding is in agreement with the observations for Schottky devices on highsymmetry GaAs surfaces [14]. This shift has a value of approximately $10 \mathrm{ps,}$ which is at the limit of our temporal resolution.

Figures 3(a) and 3(b) show the signals $\delta V(t)$ for various optical-excitation densities $W$ of the $\mathrm{Al}$ film. The first peak for QLA phonons in $V(t)$ moves to earlier times with the increase of $W$. Such behavior was observed in earlier works performed with picosecond acoustic techniques and is due to nonlinear acoustic properties of the GaAs substrate $[20,21]$. Here, for $W=6.4 \mathrm{~mJ} \mathrm{~cm}^{-2}$, the temporal shift relative to small $W$ is equal to approximately $100 \mathrm{ps}$, which is similar to the experiments when LA phonons propagate in a high-symmetry [100] direction in GaAs. In contrast to QLA, the arrival time for QTA phonons does not depend on $W$, which means that elastic nonlinear effects for QTA phonons are not so important [22].

Figures 4(a) and 4(b) show the measured $\delta V(t)$ at the elevated temperature $T=298 \mathrm{~K}$. The temporal shift corresponds to the decrease of sound velocity with the increase
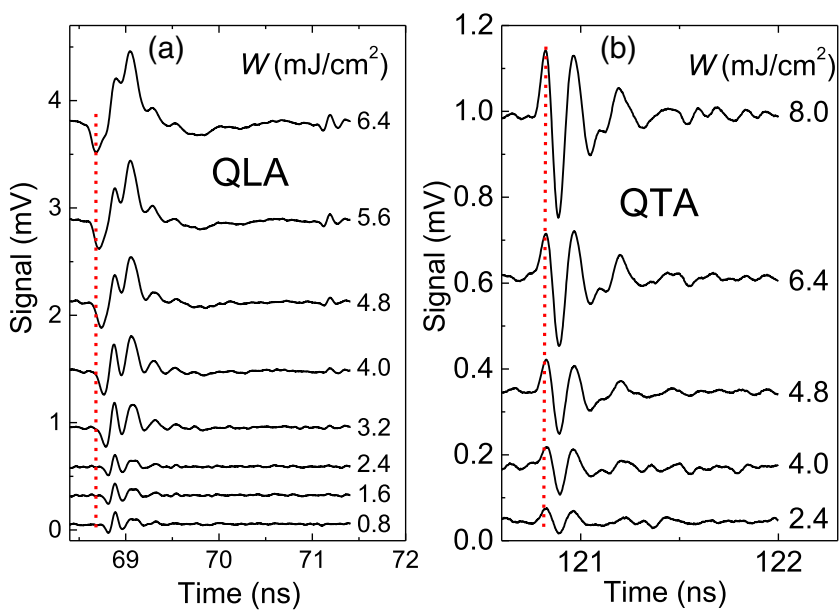

FIG. 3. Phonon-induced signals measured at zero bias, $V_{B}=0 \mathrm{~V}$, at various pump fluences for (a) QLA and (b) QTA phonons. Vertical dotted lines show the first-peak temporal shift to earlier times with the increase of $W$ for QLA, and the absence of the shift for QTA phonons. 


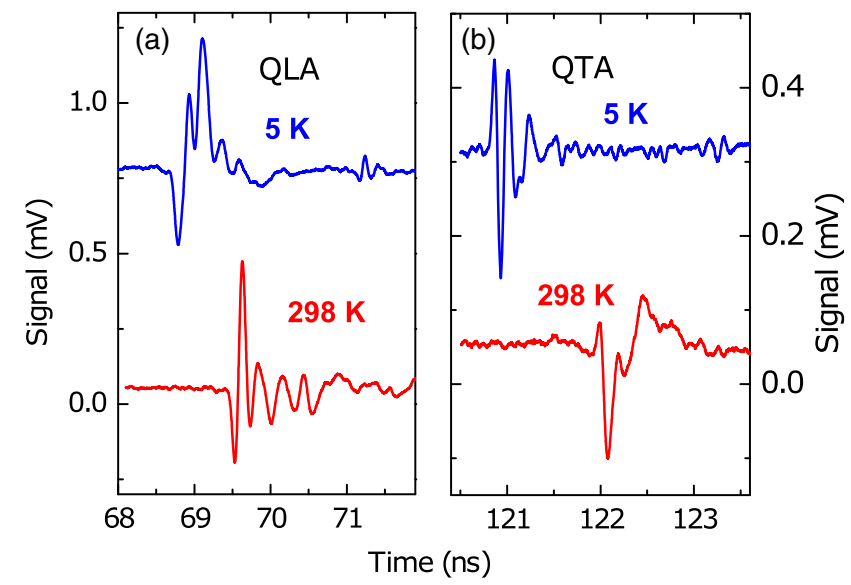

FIG. 4. Experimentally measured signals at two temperatures $T$ for (a) QLA and (b) QTA phonons.

of $T$. This approximately $1 \%$ change is in agreement with earlier measurements [23]. The amplitude of the signals does not decrease much, which is consistent with studies of the attenuation for phonons if we assume the frequencies we are sensitive to are mostly $<50 \mathrm{GHz}$ [23].

\section{ANALYSIS AND DISCUSSION}

The fact that both QLA and QTA phonons are detected in the experiments points to the importance of the PE effect in the transformation of strain to an electrical signal in the device. The GaAs conduction band is isotropic, so DP coupling to the QTA mode is expected to be very weak. The QLA mode may couple via both the DP and PE interactions. The main goal of the following analysis and discussion of the measurements is to understand the mechanisms of the electrical response of the Schottky contact to the incident coherent phonons. The currents induced by strain components $u_{z z}$ and $u_{x z}$ are the displacement currents which appear due to electron screening of the potential perturbation induced by dynamical strain [14]. This perturbation can be due to either DP or PE polarization. In our case, the characteristic frequency of coherent phonons is much less than the inverse dielectric relaxation time but much higher than $(R C)^{-1}(R$ and $C$ are the resistance and the capacitance of the diode) and then the induced voltage changes $\delta V$ on the Schottky contact may be expressed as [18]

$$
\delta V=V_{\mathrm{PE}}\left(z_{0}\right)-V_{\mathrm{PE}}\left(z_{d}\right)+\frac{\epsilon_{s}+\epsilon_{m}}{2 \epsilon_{m}} V_{\mathrm{DP}}\left(z_{0}\right)-V_{\mathrm{DP}}\left(z_{d}\right),
$$

where $V_{\mathrm{PE}}(z)$ and $V_{\mathrm{DP}}(z)$ are the strain-induced changes in the potential at a coordinate $z$ due to PE and DP polarizations, respectively; $z_{0}$ and $z_{d}$ are the coordinates of the semiconductor-metal interface and the edge of the depletion layer in the semiconductor, respectively; and $\epsilon_{s, m}$ are the lattice permittivities of the semiconductor and the metal.

In a semiconductor, $V_{\mathrm{DP}}$ is determined by the standard expression for electrons in the $\Gamma$ valley: $V_{\mathrm{DP}}=-\left(E_{1} / e\right) \nabla \cdot \mathbf{u}$, where $\mathbf{u}$ is the displacement and $E_{1} \sim-10 \mathrm{eV}$ is the DP constant of GaAs [24]. For $V_{\mathrm{PE}}$, we use the Poisson equation $\nabla^{2} V_{\mathrm{PE}}=\left(1 / \epsilon_{s} \epsilon_{0}\right) \nabla \cdot \mathbf{P}$, with the boundary condition $V_{\mathrm{PE}}(-\infty)=0$ and where $\mathbf{P}$ is PE polarization. For a DP, we get $V_{\mathrm{DP}}=-\left[E_{1} e_{z}^{(i)} /(e)\right] \eta_{i}\left(t-z / v_{i}\right)$ and, for a $\mathrm{PE}$ potential, $V_{\mathrm{PE}}=-\left[e_{\mathrm{eff}} /\left(\epsilon_{s} \epsilon_{0}\right)\right] \mu_{i}\left(t-z / v_{i}\right)$, where $e_{\mathrm{eff}}$ is the effective PE constant and $\mu_{i}^{\prime}=-v_{i} \eta_{i}$ represents the shape of the displacement in the pulse (here, the prime indicates differentiation over the argument of $\left.\mu_{i}, t-z / v_{i}\right)$. For our experimental scheme, we obtain $e_{\text {eff }}=-\left[2 e_{14} /\left(11^{3 / 2}\right)\right]\left[9 e_{z}^{(i)}+(16 / \sqrt{2}) e_{x}^{(i)}\right]$, where $e_{14}=$ $-0.16 \mathrm{C} \mathrm{m}^{-2}$ is the GaAs PE constant. It is important that the sign of the effective PE constant depends on the kind of (311) face at the Schottky contact $[(311) B$ or (311)A]. The above expression corresponds to (311) $B$, while the sign of $e_{\text {eff }}$ is reversed for (311)A.

Figure 5(a) shows the calculated temporal evolutions of $\delta V(t)$ for QLA and QTA phonons, respectively. For the DP contribution, we consider only the response corresponding to the depletion-layer edge in the semiconductor because, although the deformation constant in metals is not well known [25], available data suggests that it is smaller (in absolute value) than that of GaAs. The contributions from PE and DP mechanisms calculated separately show the PE mechanism to be dominant in $\delta V$ for both modes of phonons. This difference is much larger (approximately 20 times) for QTA phonons. The calculated response to the strain pulse extends to the $100-\mathrm{GHz}$ spectral range. The temporal signal $\delta V(t)$ shifts to earlier times, with the increase of reverse bias $V_{B}$ due to the increase of the depletion-layer width in the semiconductor.

A detailed comparison of the experimental and calculated temporal shapes and the amplitudes of $\delta V(t)$ is not possible due to the high-frequency limitations of the experimental setup. The calculated signals [Fig. 5(a)] possess a couple of oscillations with a frequency of approximately $20 \mathrm{GHz}$, which are due to the temporal shape of the strain pulses [Figs. 1(d) and 1(e)], which cannot be resolved in our experimental setup. The ringing oscillations observed experimentally have much lower (approximately 4-GHz) frequency and are due to the parasitic capacitance (approximately $0.1 \mathrm{pF}$ ) and inductance (approximately $10 \mathrm{nH}$ ) of the sample holder and bonding wires. The details of the frequency response in the experiment also depends on the bias-dependent equivalent circuit for which parameters are not known. For instance, with an increase of reverse bias, the capacitance of the Schottky contact decreases. Evidently, this decrease leads to an increase of the amplitude of the signal with an increase of negative bias (see Fig. 2). 


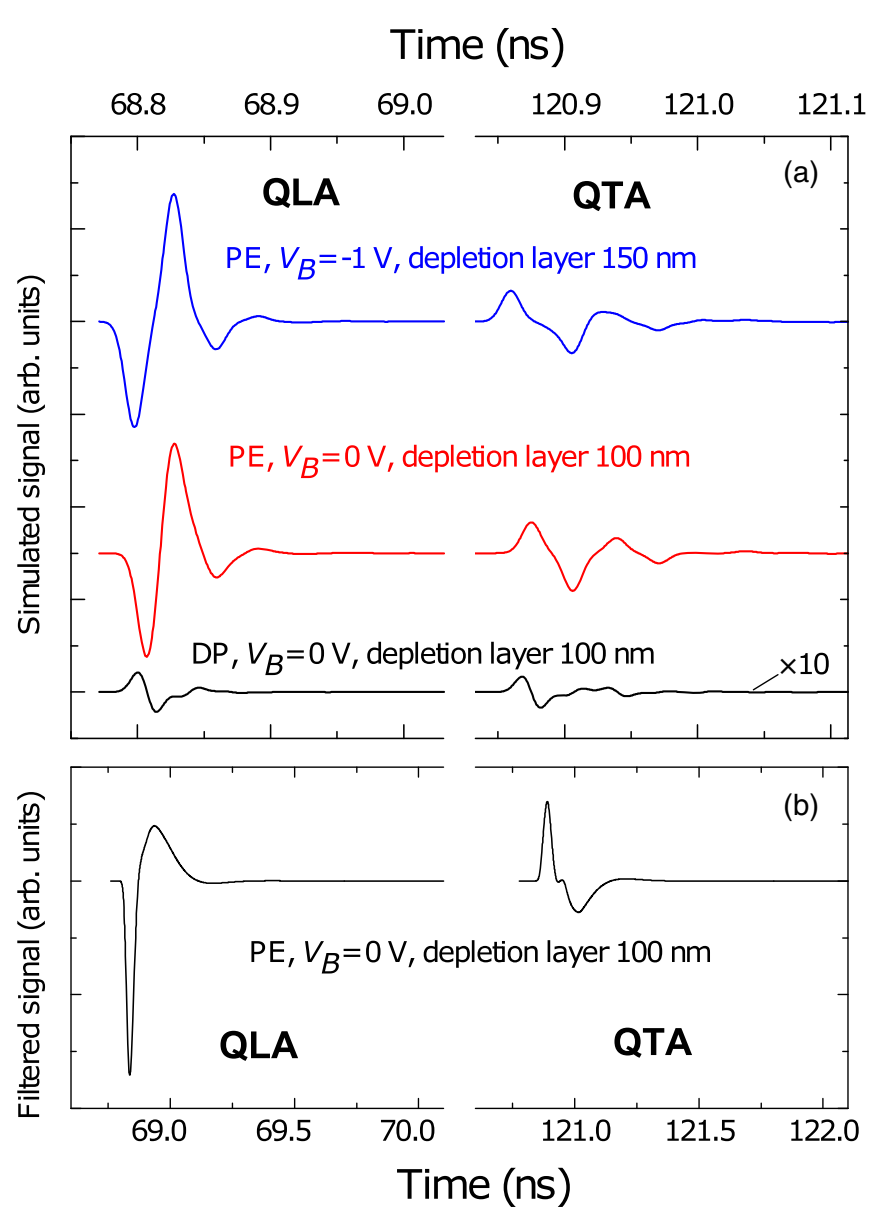

FIG. 5. (a) The theoretical signals for QLA and QTA phonons taking into account PE or DP mechanisms of phonon-electric transformation in the Schottky diode for two widths of depletion layers and corresponding bias voltage. (b) The signal after 4-GHz-cutoff low-pass filtering of the calculated signal shown in (a). Mind the different time scales in (a) and (b).

To facilitate comparison of the measured and calculated signals, we apply a low-pass filter with a cutoff at $4 \mathrm{GHz}$ to the calculated PE responses in Fig. 5(a) to account for the finite bandwidth of the experiment. The filtered responses are also shown in Fig. 5(b). This filtering does not account for the parasitic ringing in the experimental results. If we focus on the first peak in filtered $\delta V(t)$ signals in Fig. 5(b), we find agreement with the experiment for the polarity, which is negative for QLA and positive for QTA, and for the amplitude ratio (approximately 3) for the QLA and QTA first peaks. The agreement points to the predominant role of the PE phonon-electric transformation mechanism over the DP. To confirm this conclusion, we have performed similar experiments with Schottky diodes fabricated on (311)A GaAs surface and find that the polarity of the first peak in $\delta V(t)$ reverses: it becomes positive for QLA and negative for QTA phonon-induced signals. This fact is in agreement with the theoretical predictions for the PE mechanism.
The above theoretical analysis and comparison with the experimental results shows that the PE transformation from coherent phonons to the polarization is the main mechanism in the Schottky contact fabricated at (311) $B$ and (311) $A$ GaAs surfaces. This result is contrary to the case of phonon-induced electrical signals in a Schottky diode fabricated on high-symmetry (100) crystallographic planes where $\delta V(t)$ is governed exclusively by the DP mechanisms and insensitive to the shear strain components [14].

The technical limitations of the experiment do not mean that Schottky diodes cannot detect signals at much higher frequencies when using specially designed microwave holders and smaller devices. Devices with Schottky contacts a few microns in size have capacitances of order $10 \mathrm{fF}$ and have been shown to detect at frequencies up to at least $100 \mathrm{GHz}$ [26]. The acoustic signal size in these devices is comparable to that seen in this work, showing that a decrease in device size to a few microns does not significantly impact the amplitude of the detected signal.

The dominance of the PE phonon-electric transformation mechanism over DP vanishes with the increase of characteristic phonon frequency in a similar way to the matrix elements for an electron-phonon interaction [27]. Using the explicit expressions for $V_{\mathrm{DP}}$ and $V_{\mathrm{PE}}$ provided above, we can determine the threshold acoustic frequency, $f_{\text {th }}$, for which DP and PE mechanisms provide equal contributions to the Schottky-diode response and obtain $f_{\text {th }}=\left[\left(e_{\mathrm{eff}} v_{i} e\right) / 2 \pi \epsilon_{s} \epsilon_{0} E_{1} e_{z}^{i}\right]$. For QLA, $f_{\text {th }} \approx 70 \mathrm{GHz}$, while for QTA, $f_{\text {th }} \approx 250 \mathrm{GHz}$, which are higher values than the maximum of the spectrum for phonons generated in the 100-nm Al film, but they may be exceeded when phonons are generated in thinner absorbing layers or superlattices [4-8]. For higher phonon frequencies, the DP mechanism becomes dominant.

\section{CONCLUSIONS}

In this work, we show experimentally that a Schottkydiode device fabricated on a low-symmetry surface of a semiconductor serves as an efficient transducer for longitudinal and transverse coherent phonons with frequencies of about $10 \mathrm{GHz}$. Comparison of the experimental results with the theory of electron-phonon interaction reveals the dominant role of piezoelectric mechanisms in phonon-electric transformations. The theoretical calculations show that the piezoelectric mechanism dominates over deformation potential up to frequencies of 70 and $250 \mathrm{GHz}$ for longitudinal and transverse phonons, respectively. Although present experiments are limited by the gigahertz frequency range of the setup, Schottky devices fabricated in the form of micrometer mesas having low capacitance may be used as coherent phonon-electric transducers up to subterahertz frequencies. 


\section{ACKNOWLEDGMENTS}

This work was supported by the Engineering and Physical Sciences Research Council [Grant No. EP/ M016161/1] and U.S. Army Research Laboratory [Grant No. W911NF-14-1-0586].

[1] Z. L. Wang and J. Song, Piezoelectric nanogenerators based on zinc oxide nanowire arrays, Science 312, 242 (2006).

[2] J. H. Stotz, R. Hey, P. V. Santos, and K. H. Ploog, Coherent spin transport through dynamic quantum dots, Nat. Mater. 4, 585 (2005).

[3] M. T. Ong and E. J. Reed, Engineered piezoelectricity in graphene, ACS Nano 6, 1387 (2012).

[4] C.-K. Sun, J.-C. Liang, and X.-Y. Yu, Coherent Acoustic Phonon Oscillations in Semiconductor Multiple Quantum Wells with Piezoelectric Fields, Phys. Rev. Lett. 84, 179 (2000).

[5] D. M. Moss, A. V. Akimov, A. J. Kent, B. A. Glavin, M. J. Kappers, J. L. Hollander, M. A. Moram, and C. J. Humphreys, Coherent terahertz acoustic vibrations in polar and semipolar gallium nitride-based superlattices, Appl. Phys. Lett. 94, 011909 (2009).

[6] O. Matsuda, O. B. Wright, D. H. Hurley, V. E. Gusev, and K. Shimizu, Coherent Shear Phonon Generation and Detection with Ultrashort Optical Pulses, Phys. Rev. Lett. 93, 095501 (2004).

[7] O. Matsuda, O. B. Wright, D. H. Hurley, V. Gusev, and K. Shimizu, Coherent shear phonon generation and detection with picosecond laser acoustics, Phys. Rev. B 77, 224110 (2008).

[8] P. M. Walker, A. J. Kent, M. Henini, B. A. Glavin, V. A. Kochelap, and T. L. Linnik, Terahertz acoustic oscillations by stimulated phonon emission in an optically pumped superlattice, Phys. Rev. B 79, 245313 (2009).

[9] T. Pezeril, P. Ruello, S. Gougeon, N. Chigarev, D. Mounier, J.-M. Breteau, P. Picart, and V. Gusev, Generation and detection of plane coherent shear picosecond acoustic pulses by lasers: Experiment and theory, Phys. Rev. B 75, 174307 (2007).

[10] M. Lejman, G. Vaudel, I. C. Infante, P. Gemeiner, V. E. Gusev, B. Dkhil, and P. Ruello, Giant ultrafast photoinduced shear strain in ferroelectric $\mathrm{BiFeO}_{3}$, Nat. Commun. 5, 4301 (2014).

[11] Y.-C. Wen, T.-S. Ko, T.-C. Lu, H.-C. Kuo, J.-I. Chyi, and C.-K. Sun, Photogeneration of coherent shear phonons in orientated wurtzite semiconductors by piezoelectric coupling, Phys. Rev. B 80, 195201 (2009).

[12] G. D. Sanders and C. J. Stanton, Carrier dynamics and coherent acoustic phonons in nitride heterostructures, Phys. Rev. B 74, 205303 (2006).
[13] A. V. Akimov, A. V. Scherbakov, D. R. Yakovlev, C. T. Foxon, and M. Bayer, Ultrafast Band-Gap Shift Induced by a Strain Pulse in Semiconductor Heterostructures, Phys. Rev. Lett. 97, 037401 (2006).

[14] D. M. Moss, A. V. Akimov, B. A. Glavin, M. Henini, and A. J. Kent, Ultrafast Strain-Induced Current in a GaAs Schottky Diode, Phys. Rev. Lett. 106, 066602 (2011).

[15] X. Wang, J. Song, J. Liu, and Z. L. Wang, Direct-current nanogenerator driven by ultrasonic waves, Science 316, 102 (2007).

[16] C. Thomsen, H. T. Grahn, H. J. Maris, and J. Tauc, Surface generation and detection of phonons by picosecond light pulses, Phys. Rev. B 34, 4129 (1986).

[17] A. V. Scherbakov, M. Bombeck, J. V. Jäger, A. S. Salasyuk, T. L. Linnik, V. E. Gusev, D. R. Yakovlev, A. V. Akimov, and M. Bayer, Picosecond opto-acoustic interferometry and polarimetry in high-index GaAs, Opt. Express 21, 16473 (2013).

[18] B. A. Glavin, High-frequency acoustic wave detection in Schottky diodes: Theory consideration, arXiv:1607.04922.

[19] Z. V. Popović, J. Spitzer, T. Ruf, M. Cardona, R. Nötzel, and K. Ploog, Folded acoustic phonons in GaAs/AlAs corrugated superlattices grown along the [311] direction, Phys. Rev. B 48, 1659 (1993).

[20] E. Péronne and B. Perrin, Generation and detection of acoustic solitons in crystalline slabs by laser ultrasonics, Ultrasonics 44, e1203 (2006).

[21] A. V. Scherbakov, P. J. S. van Capel, A. V. Akimov, J. I. Dijkhuis, D. R. Yakovlev, T. Berstermann, and M. Bayer, Chirping of an Optical Transition by an Ultrafast Acoustic Soliton Train in a Semiconductor Quantum Well, Phys. Rev. Lett. 99, 057402 (2007).

[22] J. V. Jäger, A. V. Scherbakov, T. L. Linnik, D. R. Yakovlev, M. Wang, P. Wadley, V. Holy, S. A. Cavill, A. V. Akimov, A. W. Rushforth, and M. Bayer, Picosecond inverse magnetostriction in galfenol thin films, Appl. Phys. Lett. 103, 032409 (2013).

[23] W. Chen, H. J. Maris, Z. R. Wasilewski, and S.-I. Tamura, Attenuation and velocity of $56 \mathrm{GHz}$ longitudinal phonons in gallium arsenide from 50 to $300 \mathrm{~K}$, Philos. Mag. B 70, 687 (1994).

[24] K. Lee, M. S. Shur, T. J. Drummond, and H. Morkoq, Low field mobility of 2- $d$ electron gas in modulation doped $\mathrm{Al}_{x} \mathrm{Ga}_{1-x} \mathrm{As} / \mathrm{GaAs}$ layers, J. Appl. Phys. 54, 6432 (1983).

[25] P. J. van Hall, Ultrafast processes in $\mathrm{Ag}$ and $\mathrm{Au}$ : $\mathrm{A}$ Monte Carlo study, Phys. Rev. B 63, 104301 (2001).

[26] S. L. Heywood, B. A. Glavin, R. P. Beardsley, A. V. Akimov, M. W. Carr, J. Norman, P. C. Norton, B. Prime, N. Priestley, and A. J. Kent, Heterodyne mixing of millimetre electromagnetic waves and sub- $\mathrm{THz}$ sound in a semiconductor device, Sci. Rep. 6, 30396 (2016).

[27] Carrier Scattering in Metals and Semiconductors, Vol. 19, edited by V. F. Gantmakher and Y. B. Levinson (Elsevier, New York, 2012). 